Now $K$ and $J$, increased by unity, give (apart from a multiple of 3 ) the number of sets of values for which a cubic form (with the coefficients not all zero) vanishes in the $G F[3]$ and the $G F\left[3^{2}\right]$, respectively. We find that *

$$
\begin{aligned}
J & =K+\Delta^{2}-\Delta \quad(\Delta=\text { discriminant }), \\
K^{2}+K & =J^{2}+J
\end{aligned}
$$

But $K$ is not a rational function of $J$ (in view of the first and second forms below), nor $J$ a rational function of $K$ (in view of the second and third forms) :

\begin{tabular}{c|r|r|r} 
Form. & $K$ & $J$ & \multicolumn{1}{|c}{} \\
\hline$x^{3}-x y^{2}+y^{3}$ & -1 & -1 & 1 \\
$x^{3}+x y^{2}$ & 0 & -1 & -1 \\
$x^{3}$ & 0 & 0 & 0 \\
$x^{2} y+x y^{2}$ & -1 & -1 & 1 \\
$x^{2} y$ & 1 & 1 & 0 \\
Vanishing & 0 & 0 & 0
\end{tabular}

Every cubic can be transformed modulo 3 into one of those given in the table (Transactions, 1. c., page 232).

The University of Chicago, January, 1908.

\title{
NOTE ON JACOBI'S EQUATION IN THE CALCULUS OF VARIATIONS.
}

BY PROFESSOR MAX MASON.

(Read before the American Mathematical Society, February 29, 1908.)

Is Weierstrass's theory of the calculus of variations $\dagger$ it is shown that the determinant

$$
\omega=\frac{\partial y}{\partial t} \frac{\partial x}{\partial a}-\frac{\partial x}{\partial t} \frac{\partial y}{\partial a}
$$

formed from the equations $x=x(t, a), y=y(t, a)$ of a family of extremals of the integral

\footnotetext{
* If we employ the invariant $P=\Delta+1-K$ (1. c., p. 211), we have $J=K^{2}+K+P-1$. 1904.

† See for example Bolza, Lectures on the calculus of variations, Chicago,
} 


$$
J=\int F\left(x, y, x^{\prime}, y^{\prime}\right) d t
$$

is a solution of Jacobi's equation

$$
\left(\omega^{\prime} F_{1}\right)^{\prime}-\omega F_{2}=0 .
$$

This result, which is of fundamental importance in the theory, is obtained by differentiating the Euler equations of the extremals

$$
F_{x}-\frac{d}{d t} F_{x^{\prime}}=0, \quad F_{y}-\frac{d}{d t} F_{y^{\prime}}=0
$$

with respect to the parameter $a$, a method which involves considerable reckoning and the introduction of two sets of functions $L, M, N ; L_{1}, M_{1}, N_{1}$, which serve to define $F_{2}$.

It is the object of this note to derive the result above stated directly from the single equation of the extremals

$$
T \equiv F_{x y^{\prime}}-F_{y x^{\prime}}+F_{1}\left(x^{\prime} y^{\prime \prime}-y^{\prime} x^{\prime \prime}\right)=0,
$$

which is equivalent to the pair of dependent Euler equations. The introduction of successive sets of auxiliary functions to define $F_{2}$ is in this way avoided, and an explicit form for $F_{2}$ is obtained.

Write for abbreviation $\partial x / \partial a=\xi, \partial y / \partial a=\eta$, and denote differentiation with respect to $t$ by accents. Then

$$
\begin{gathered}
\omega=y^{\prime} \xi-x^{\prime} \eta, \quad \omega^{\prime}=y^{\prime \prime} \xi-x^{\prime \prime} \eta+y^{\prime} \xi^{\prime}-x^{\prime} \eta^{\prime}, \\
\omega^{\prime \prime}=y^{\prime \prime \prime} \xi-x^{\prime \prime \prime} \eta+2\left(y^{\prime \prime} \xi^{\prime}-x^{\prime \prime} \eta^{\prime}\right)+y^{\prime} \xi^{\prime \prime}-x^{\prime} \eta^{\prime \prime} .
\end{gathered}
$$

If equation (1) be differentiated with respect to $a$, and the quantity $\left[y^{\prime \prime \prime} \xi-x^{\prime \prime \prime} \eta+3\left(y^{\prime \prime} \xi^{\prime}-x^{\prime \prime} \eta^{\prime}\right)\right] F_{1}$ be subtracted and added in the result, the following equation is obtained :

$$
\begin{aligned}
-\omega^{\prime \prime} & F_{1}+\xi^{\prime}\left[3 y^{\prime \prime} F_{1}+\left(x^{\prime} y^{\prime \prime}-y^{\prime} x^{\prime \prime}\right) F_{1 x^{\prime}}-x^{\prime} y^{\prime} F_{1 x}-y^{\prime 2} F_{1 y}\right] \\
& +\eta^{\prime}\left[-3 x^{\prime \prime} F_{1}+\left(x^{\prime} y^{\prime \prime}-y^{\prime} x^{\prime \prime}\right) F_{1 y^{\prime}}+x^{\prime 2} F_{1 x}+x^{\prime} y^{\prime} F_{1 y}\right] \\
& +\xi\left[y^{\prime \prime \prime} F_{1}+\left(x^{\prime} y^{\prime \prime}-y^{\prime} x^{\prime \prime}\right) F_{1 x}+F_{x x y^{\prime}}-F_{x y x^{\prime}}\right] \\
& +\eta\left[-x^{\prime \prime \prime} F_{1}+\left(x^{\prime} y^{\prime \prime}-y^{\prime} x^{\prime \prime}\right) F_{1 y}+F_{x y y^{\prime}}-F_{y y x^{\prime}}\right]=0 .
\end{aligned}
$$

Since

$$
F_{1}^{\prime}=x^{\prime \prime} F_{1 x^{\prime}}+y^{\prime \prime} F_{1 y^{\prime}}+x^{\prime} F_{1 x}+y^{\prime} F_{1 y},
$$

the coefficients of $\xi^{\prime}$ and $\eta^{\prime}$ are equal to 


$$
\begin{array}{r}
y^{\prime \prime}\left(3 F_{1}+x^{\prime} F_{1 x^{\prime}}+y^{\prime} F_{1 y^{\prime}}\right)-y^{\prime} F_{1}^{\prime}, \\
-x^{\prime \prime}\left(3 F_{1}+x^{\prime} F_{1 x^{\prime}}+y^{\prime} F_{1 y^{\prime}}\right)+x^{\prime} F_{1}^{\prime \prime},
\end{array}
$$

respectively. Now it may be shown from the homogeneity property of $F$ that

$$
3 F_{1}+x^{\prime} F_{1 x^{\prime}}+y^{\prime} F_{1 y^{\prime}}=0 .
$$

In fact, on differentiating the identity

$$
x^{\prime} F_{x^{\prime}}+y^{\prime} F_{y^{\prime}}=F
$$

twice with respect to $x^{\prime}$, the equation

$$
F_{x^{\prime} x^{\prime}}+x^{\prime} F_{x^{\prime} x^{\prime} x^{\prime}}+y^{\prime} F_{x^{\prime} x^{\prime} y^{\prime}}=0
$$

is obtained. If the second derivatives be expressed in terms of $F_{1}$, this equation becomes

$$
y^{\prime^{2}}\left(3 F_{1}+x^{\prime} F_{1 x^{\prime}}+y^{\prime} F_{1 y^{\prime}}\right)=0 .
$$

A similar equation, where the factor $y^{\prime 2}$ is replaced by $x^{\prime 2}$, is obtained by differentiating (4) with respect to $y^{\prime}$. Since $x^{\prime}$ and $y^{\prime}$ are not simultaneously zero, equation (3) must hold. The coefficients of $\xi^{\prime}$ and $\eta^{\prime}$ in equation (2) are therefore $-y^{\prime} F^{\prime}$ and $x^{\prime} F_{1}^{\prime}$ respectively. After adding and subtracting the expression $\left(y^{\prime \prime} \xi-x^{\prime \prime} \eta\right) F_{1}^{\prime}$, equation (2) takes the form

$$
-\left(\omega^{\prime} F_{1}\right)^{\prime}+P \xi+Q \eta=0,
$$

where

$$
\begin{aligned}
& P=y^{\prime \prime \prime} F_{1}+\left(x^{\prime} y^{\prime \prime}-y^{\prime} x^{\prime \prime}\right) F_{1 x}+F_{y^{\prime} x x}-F_{x^{\prime} x y}+y^{\prime \prime} F_{1}^{\prime}, \\
& Q=-x^{\prime \prime \prime} F_{1}+\left(x^{\prime} y^{\prime \prime}-y^{\prime} x^{\prime \prime}\right) F_{1 y}+F_{y^{\prime} x y}-F_{x^{\prime} y y}-x^{\prime \prime} F_{1}^{\prime} .
\end{aligned}
$$

Now

$$
x^{\prime} P+y^{\prime} Q=\frac{d}{d t} T=0
$$

so that there exists a function $F_{2}$ such that

$$
P=y^{\prime} F_{2}, \quad Q=-x^{\prime} F_{2} .
$$

Therefore, after changing the signs in equation (5) the desired equation

is obtained.

$$
\left(\omega^{\prime} F_{1}\right)^{\prime}-\omega F_{2}=0
$$


The function $F_{2}$ determined by equations (6) and (7) may be found explicitly from the equation

$$
\left(x^{\prime 2}+y^{\prime 2}\right) F_{2}=y^{\prime} P-x^{\prime} Q \text {. }
$$

On expanding the second member and collecting terms, this equation becomes

$$
\begin{aligned}
\left({x^{\prime}}^{2}+y^{\prime 2}\right) F_{2}= & \left(x^{\prime} x^{\prime \prime \prime}+y^{\prime} y^{\prime \prime \prime}\right) F_{1}+\left(x^{\prime} x^{\prime \prime}+y^{\prime} y^{\prime \prime}\right) F_{1}^{\prime} \\
& -F_{x x^{\prime}}^{\prime}-F_{y y^{\prime}}^{\prime \prime}+x^{\prime}\left(F_{x^{\prime} x x}+F_{x^{\prime} y y}\right)+y^{\prime}\left(F_{y^{\prime} x x}+F_{y^{\prime} y y}\right) .
\end{aligned}
$$

Now on differentiating the identity

$$
x^{\prime} F_{x^{\prime}}+y^{\prime} F_{y^{\prime}}=F
$$

twice with respect to $x$ or $y$, the equations

$$
x^{\prime} F_{x^{\prime} x x}+y^{\prime} F_{y^{\prime} x x}=F_{x x}, \quad x^{\prime} F_{x^{\prime} y y}+y^{\prime} F_{y^{\prime} y y}=F_{y y}
$$

are obtained, so that $F_{2}$ is given by the equation

$$
\begin{aligned}
\left(x^{\prime 2}+y^{\prime 2}\right) F_{2}=\left(x^{\prime} x^{\prime \prime \prime}\right. & \left.+y^{\prime} y^{\prime \prime \prime}\right) F_{1} \\
& +\left(x^{\prime} x^{\prime \prime}+y^{\prime} y^{\prime \prime}\right) F_{1}^{\prime}+F_{x x}-F_{x x^{\prime}}^{\prime}+F_{y y}-F_{y y^{\prime}}^{\prime}
\end{aligned}
$$

In case the parameter $t$ is the length of arc, so that $x^{\prime 2}+y^{\prime 2} \equiv 1$, the function $F_{2}$ has the simpler form

$$
\begin{aligned}
& F_{2}=F_{x x}-F_{x x^{\prime}}^{\prime}+F_{y y}-F_{y y^{\prime}}^{\prime}-\left(x^{\prime \prime 2}+y^{\prime \prime 2}\right) F_{1} . \\
& \text { SheFfield Scinemific School, } \\
& \text { YaLe UnIVERSITY. }
\end{aligned}
$$

\section{ON THE DISTANCE FROM A POINT TO A SURFACE.}

BY PROFESSOR E. R. HEDRICK.

(Read before the Anerican Mathematical Society, September 5, 1907.)

The discussion of the extrema of the distance from a point to a surface has been made the basis for the treatment of principal radii of curvature and for the classification of points on a surface by several writers.* In this connection it is interest-

* See, e. g., Goursat, Cours d'analyse, or English translation, no. 60 ; the statements there made are correct, the example here considered falling under the case $s^{2}-r t=0$. See also Bulletin, vol. 13 , no. 9, pp. 447, 448 ; the statements of this article differ in their spirit from those of the present article, and comparisons must be made with this understanding. 